\title{
Profile of Caesarean Section at Kathmandu Medical College
}

\author{
Apurwa Prasad, ${ }^{1}$ Garima Bhandari, ${ }^{1}$ Rachana Saha ${ }^{1}$ \\ ${ }^{1}$ Department of Obstetrics and Gynecology, Kathmandu Medical College, Kathmandu, Nepal
}

\begin{abstract}
Background: There is a world-wide rise in caesarean section rateduring the last three decades and has been a cause of alarm and needs an in-depth study. The objective of this study was to determine the rate and clinical indications of Caesarean Section.

Methods: A hospital based study was carried out from 15th June 2015 to 15th January 2016 in Department of Obstetrics and Gynecology at Kathmandu Medical College, Sinamangal, Nepal. Patients who delivered by caesarean section were included in the study. Basic demographic data and clinical indications were noted.

Results: A total of 1172 deliveries were carried out during the study period. Total number of caesarean section was 537 accounting to $45.81 \%$. Most of the patients were of the age group of 25-29 years (42.8\%). Most of the patients were primigravida $(\mathrm{n}=274 ; 51 \%)$. Emergency caesarean section was $411(76.5 \%)$ and elective caesarean section was 126 (23.4\%). Multigravida (71\%) underwent more elective procedure than primigravida (25.39\%). The most frequent indication was fetal distress $19.55 \%(\mathrm{n}=105)$, failed induction $19.73 \%(\mathrm{n}=106)$, and previous caesarean section $21.3 \%$ $(\mathrm{n}=115)$.

Conclusions: The rate of cesarean section is quite high than that recommended by WHO which is (10-15\%). Most of the caesarean sections were emergency caesarean section with previous caesarean being the leading cause.

Keywords: Caesarean section; indication; rate of caesarean section.
\end{abstract}

\section{INTRODUCTION}

Cesarean section rate (CSR) has been a public health concern since last few years. ${ }^{1-12}$ Increase in CSR has been attributed to factors such as fetal monitoring with early detection of fetal distress, need for repeated cesarean section (CS), advancing maternal age at delivery, socioeconomic factors and changes in clinical management of labor. ${ }^{1-12}$ Although CSR in many countries have increased from recommended level in both developed and many developing countries with increasing institutional deliveries due to growing access to gynecological and obstetrical care, the rate of delivery through cesarean section is relatively low in Nepal. ${ }^{9}$ However, some earlier studies from Nepal on hospital based data shows that cesarean section deliveries are increasing rapidly in eastern part of Nepal. ${ }^{4}$ This present study was conducted to find out the rate and indication in our set up. This may be of help in adapting suitable measure to reduce CSR and problems associated with it.

\section{METHODS}

A hospital based cross-sectional study was conducted in the department of Obstetrics and Gynecology, Kathmandu Medical College. All the patients undergoing CS from the $15^{\text {th }}$ June 2015 to $15^{\text {th }}$ January 2016 were included in the study.

Before starting the study, ethical approval of the study was obtained from the institutional review board of Kathmandu Medical College. Informed consent was obtained from the patient and their participation in the study was voluntary. The participants were enrolled from the in-ward patients of Department of Gynaecology and Obstetrics. Demographic and clinical data were recorded in detailed proforma.

Elective cesarean section was defined as those performed without emergencies and decision was made before the onset of labour. Emergency cesarean section were defined as those performed for emergency fetomaternal reasons. Clinical indication was noted for both 
Profile of Caesarean Section at Kathmandu Medical College

elective and emergency cesarean section. The period of gestation at the time of presentation, gravidity, time of presentation, parity and obstetrics, background was noted.

The completed proforma was handled with discretion and collected information was accessible only to those directly involved in research. The data was entered and analyzed using Microsoft Excel. The CSR was calculated as the number of CS per 100 deliveries. The percentage for each emergency and elective cesarean section was calculated. Proportions of indication specific cesarean section were calculated as the number of indicationspecific cesarean deliveries per 100 cesarean sections. Results were presented as proportions and percentage.

\section{RESULTS}

Total deliveries were 1172.The CSR was 45.81\% (Table 1).

\section{Table 1. Type of Delivery $(n=1172)$.}

\section{Types of deliveries}

Normal delivery

No of Patients

(\%)

$593(50.59 \%)$

Instrumental delivery

$23(1.96 \%)$

Caesarean section

537 (45.81\%)

Others (IUFD, Breech, Still birt )

$19(1.62 \%)$

All the patients delivered were in the age group of 15 39 years. Most of the patients delivered were in the age group of $25-29$ years $(n=230 ; 42$. 8\%). Advanced maternal age undergoing CS were only $5 \%$. Primigravida $(n=74 ; 51.02 \%)$ underwent CS more than multigravida. Multigravida $(n=92 ; 73.8 \%)$ underwent more elective CS than primigravida (Table 2).

\section{Table 2. Category of caesarean section $(n=537)$.}

Mode of caesarean Section

No. of Patients (\%)

Elective CS

$126(23.4 \%)$

Emergency CS

$411(76.5 \%)$

Various indications of CS are shown in Table 3. The most common indication was failed induction, fetal distress, previous LSCS and previous LSCS including other obstetric risk factor respectively.
Table 3. Indications of caesarean section $(n=537)$.

Indications

Total Patients (\%)

Failed induction

$106(19.73 \%)$

Fetal distress

$105(19.55 \%)$

Previous LSCS

$62(11.5 \%)$

Pervious LSCS with other risk

factors

CPD

$58(10.8 \%)$

Meconium stained liquor

$52(9.6 \%)$

Breech/ Malpresentation

$46(8.5 \%)$

Hypertensive disorder

$15(2.7 \%)$

Oligohydraminous

$12(2.22 \%)$

Multiple pregnancy

$11(2.04 \%)$

$\mathrm{APH}$

$6(1.11 \%)$

NPOL

DTA

Others

$5(0.9 \%)$

APH: ante -partum hemorrhage; NPOL: non-progress of labor; DTA: deep transverse arrest.

\section{DISCUSSION}

The increasing rate of CS is not only in Nepal but all over the world and is an area of major concern. ${ }^{1-12}$ There might be various factors associated involved in the high $\mathrm{CSR}^{3}{ }^{3}$ It may be due to fear of litigation, health insurance system, CS by choice, increased use of electronic monitoring of fetus, and increased proportion of breech deliveries by CS and deliveries in private set up. ${ }^{1,3,6,10-12}$

Our CSR was $45.81 \%(n=537)$ which is quite high when compared to similar studies done in Eastern Nepal by Chhetri et $\mathrm{al}^{4}$ in 2007 of $33.7 \%$. They also commented that increment was of $5 \%$ from the year 2006. Subediet al., ${ }^{5}$ recorded $19.89 \%$ in year 2011 . The prevalence of CS delivery has increased by more than four times from 2001 to $2011 .{ }^{9}$ They also concluded that older women of age, urban, being educated, having educated partner and being rich were the major characteristic of the mother who had caesarean delivery. ${ }^{9}$ In China, there is single child policy but the CSR is high which is surprising given the lack of the factors that led to high CSR is 
not there. ${ }^{1}$ The high CSR be an early indication that emerging forms of health insurance system and fee for service payments to physicians will lead to an excessive emphasis on costly, high technology medical care in China. ${ }^{1}$ Our hospital has adopted the Aama programme of the safe motherhood program of Government of Nepal which promotes institutional delivery and makes the delivery charges free including the complications. Patients are not given the autonomy of decision making but the health personnel play the key role in making the decisions similar in context to, Shamshad et al. ${ }^{3}$ The right to choose CS involves many issues. The relative safety of an elective CS in developed world has given to another controversy. However, CS on demand threatens national resources, and is an expensive and dangerous luxury. ${ }^{1,6}$ FIGO states that performing CS for nonmedical reasons are not justified. ${ }^{6}$ The fear of litigation cannot be ruled out. Defensive obstetrics is another common reason for high rates of CS. Defensive obstetrics violates the fundamental principle of medical practice though it has grown in numbers but there has been no slowdown in litigation. ${ }^{6}$ Health insurance system has minimal role in our set up. Our hospital acts as tertiary level referral centre and high-risk cases are received for better maternal and fetal outcome which warrants CS.

The most common reasons for performing CS were previous CS (21.3\%), failed induction (19.73\%) and fetal distress(19.55\%). The most frequent indication was previous CS as it is all over the world. However, current medical literature suggests that $60-80 \%$ women can achieve vaginal delivery. ${ }^{7}$ Sharma et al in their study found indication of repeat elective caesarean section was refusal for trial of $\operatorname{scar}(n=15 ; 43.8 \%) .{ }^{7}$ When the women were told about the risk associated with trial of scar like uterine rupture, scar dehiscence and bleeding due to morbidly adherent placenta, they did not consent for trial of scar and opted for elective CS. An obstetrician find repeat caesarean section much safer and convenient and is less likely to give rise to complication of scar dehiscence and possible subsequent litigation and maternal preference. ${ }^{3-6}$ It is evident that where as CS is doctor friendly, VBAC is not. ${ }^{6}$ Our institute may have similar reasons for practicing in cases of previous CS.

Failed induction rate was as high as $19.73 \%(n=106)$. The current research suggests that labor induction makes CS more likely among first time when cervix is unfavorable. ${ }^{3,12}$ Unjustified induction with prostaglandins without prior assessment of risk factors, fetal size, presentation, stage of labor ,position and pelvic adequacy makes induction fail. ${ }^{12} \mathrm{~A}$ longer intrapartum course leads to overutilization of resources, obstructed labor, maternal and fetal distress, and ruptured uterus. Proper evaluation and patient plays a key role in decision making and unnecessary intervention. ${ }^{3}$ The search of new cervical priming agent is continued as Cerviprime (Prostaglandin $\mathrm{E}_{1}$ ) was out of market and was not available and tablet Mifepristone was used as new cervical ripening agent. All the patients were induced at 41 weeks as per departmental policy.

Fetal distress was third leading cause of caesarean section. Fetal distress was diagnosed by fetal heart rate and frequent use of cardio-tocograph to monitor the fetal heart rate as admission test and in suspicious cases of fetal distress diagnosed during intermittent auscultations. Similar scenario was cited by Chhetri et al. ${ }^{4}$ The diagnosis of fetal distress is often subjective and lacks standard clinical criteria in different health facilities. ${ }^{3,10}$ Cardiotocographic monitoring is known to overestimate the fetal distress. ${ }^{4,10}$ Many gestational and antepartum factors and uteroplacental vascular disease, reduced uterine perfusion and cord compression can be singularly or in combination influence the fetal response in a CTG. ${ }^{10}$ Methods of screening and diagnosing the condition have thus limitation. The accurate method for establishment of fetal distress is to perform fetal scalp blood pH estimation, which is considered the gold standard for the assessment of fetal wellbeing but is not performed in our set up. Similar practice has been in this part of world..$^{3-5,10}$

Meconium stained liquor was $9.6 \%$ and was to prevent meconium aspiration syndrome which is an important cause of neonatal morbidity and mortality. Due to unavailability gold standard of diagnosing fetal distress with scalp $\mathrm{pH}$ is unavailable, hence CS rate is high. Similar reasons have been cited for high CSR. ${ }^{3-5,10-12 .}$

Hannah et al in their meta-analysis showed significantly lower rates of perinatal morbidity and mortality with planned caesarean section than with planned vaginal birth in cases of breech. ${ }^{16}$ Our departmental policy is to practice planned CS for all breech until and unless they come late in labor. In some countries CSR for breech is now the order of $80 \% .^{3-4}$ This trend has implications not for the index pregnancy but increases the chance of repeat CS in subsequent pregnancy. ${ }^{3}$ Practice of external cephalic version can reduce the likelihood of $\mathrm{CS}^{3,10}$

\section{CONCLUSIONS}

The rate of CS is quite high. The most common indications were previous CS, fetal distress, failed induction, meconium stained liquor, and breech deliveries. 


\section{REFERENCES}

1. Cai WW, Marks JS, Chen CH, Zhuang YX, Morris L, Harris JR. Increased cesarean section rates and emerging patterns of health insurance in Shanghai, China. Am J Public Health. 1998;88(5):777-80. [Link]

2. Al-Mulhim A, Abu-Heija AT, Ali AHM, Bahnasy A. Factors Affecting the Rates and the Indicators of Primary Caesarean Section. Bahrain Med Bull. 2001;23(4)160-62. [Full Text]

3. Shamshad. Factors leading to increased cesarean section rate. Gomal J Med Sci. 2008;6(1):1-4. [Full Text]

4. Chhetri S, Singh U. Caesarean section: its rate and indications at tertiary referral centre in Eastern Nepal. J Med Science. 2011;9(3):179-83. [Link]

5. Subedi S. Rising rate of caesarean section- a year review. J Nobel Med Coll. 2012;1(2):72-6. [Full Text]

6. Mukherjee SN. Rising caesarean rate. J ObstetGynecol India. 2006;56(4):298-300. [Full Text]

7. Sharma A, Sharma U, Chaudhary P, Acharya A, Chaudhary A, Hanspal J. Maternal and neonatal outcome in patients with history of previous one caesarean section. Indian Medical Gazette. 2012;145(5):169-73. [Full Text]

8. Saha S, Saha S, Das R, Chakraborty M, Bala HS, Naskar P. A paradigm shift to check the increasing trend of cesarean delivery is the need to hour: but how? J ObstetGynecolIndia. 2012;62(4):391-7. [PubMed]

9. KC P, Neupane S. Caesarean deliveries among Nepalese mother: changes over time 2001-2011 and determinants. Arch Gynecol Obstet. 2014;289(2):421-7. [PubMed]

10. Unnikrishnan B, Rakshith P, Aishwarya A, Nithin K, Rekha T, Prasanna P, et al. Trends and Indications for Caesarean
Section in a tertiary care Obstetric Hospital in Coastal South India. AMJ. 2010;3(2):821-5. [Full Text]

11. Naeem M, Khan MZ, Abbas SH, Khan A, Adil M, Khan MU. Rate and indication of elective and emergency caesarean section; a study in a tertiary care hospital of Peshawar.J Ayub Med Coll Abbottabad. 2015;27(1):151-4. [PubMed]

12. Hafeez M, Yasin A, Badar N, Pasha MI, Akram N, Gulzar B. Prevalence and Indications of Caesarean Section in a Teaching Hospital. JIMSA. 2014;27(1):15-6. [Full Text]

13. Appropriate technology for birth. Lancet. 1985;2(8452):436-7. [PubMed]

14. Chalmers B. Appropriate technology for birth revisited. Br J Obstet Gynaecol. 1992;99(9):709-10. [PubMed]

15. Leitch CR, Walker JJ. Cesarean section rates. Evaluate the reasons for surgery. BMJ. 1994;308:133-4. [PubMed]

16. Hannah M, Hannah W. Caesarean section or vaginal birth for breech presentation at term. BMJ. 1996 Jun;312:14334. [PMC] 